\title{
La Agenda 2030 como una respuesta a la crisis global del desarrollo sostenible: potencialidades y límites*
}

\section{The 2030 Agenda as a model to deal with the global crisis of sustainable development: potentialities and limits}

\section{Natalia Millán Acevedo**}

Artículo de reflexión

Fecha de recepción: 29 de marzo de 2021

Fecha de aceptación: 4 de junio de 2021

\section{Para citar este artículo:}

Millán Acevedo, N. (2021). La Agenda 2030 como una respuesta a la crisis global del desarrollo sostenible: potencialidades y límites. Revista Análisis Jurídico-Político, 3(6), 55-73. https:// doi.org/10.22490/26655489.4590

\section{Resumen}

En un mundo interdependiente y transnacionalizado — que se ha configurado como una verdadera sociedad del riesgo global一, la Agenda 2030 para el Desarrollo Sostenible podría marcar un punto de inflexión en el trabajo por el desarrollo al proponer metas y objetivos que todos los países debieran seguir para construir un mundo justo, sostenible y cohesionado. En este marco, el presente artículo

\footnotetext{
* Este artículo de reflexión se realizó en el marco del proyecto de investigación titulado Articulación de Agendas globales y Agendas nacionales: el proceso de implementación de la Agenda 2030 en Europa y América Latina, con referencia: PID2019-104967RB-I00, concedido por el Plan Nacional de I+D+I del Ministerio de Ciencia e Innovación. Universidad Complutense de Madrid. Período: 2020-2023.

** Doctora en Ciencia Política y profesora del Departamento de Ciencia Política y de la Administración de la Facultad de Ciencia Política y Sociología de la Universidad Complutense de Madrid. Ha sido profesora de la Universidad Pontificia Comillas y actualmente es profesora visitante de diversos másteres y doctorados en temas de desarrollo sostenible. Sus ámbitos de investigación se relacionan con la coherencia de políticas públicas para el desarrollo, la globalización y sus impactos en las políticas locales, y la teoría política del desarrollo. Correo electrónico: nmillana@ucm.es; ORCID: https://orcid.org/0000-0001-8310-8532
} 
pretende analizar la Agenda 2030 para comprehender las oportunidades, dificultades y contradicciones que plantea en cuanto a la promoción del desarrollo humano y la sostenibilidad ambiental. El artículo argumenta que, si bien la agenda presenta importantes límites que se relacionan con la falta de cuestionamiento sobre las relaciones de poder hegemónicas y estructurales, se trata de una verdadera oportunidad para colocar en el centro del sistema político y económico al desarrollo sostenible, y dar respuesta a la crisis multidimensional a la que se enfrenta la humanidad.

Palabras clave: Agenda 2030, desarrollo sostenible, interdependencia, crisis multidimensional.

\section{Abstract}

We live in an interdependent and transnationalized world - that has been configured as a global risk society. In this framework-, the 2030 Agenda for Sustainable Development could mark a turning point in development work by proposing goals and objectives that all countries should follow to build a more sustainable world. With this framework, this article aims to carry out an analysis of the 2030 Agenda to understand its opportunities, difficulties, and contradictions. The article argues that although the Agenda presents important limitations that are related to the lack of questioning structural power relations, it is a true opportunity to place sustainable development at the centre of the political and economic system and to develop policies to deal with the multidimensional crisis that affects all societies.

Keywords: Agenda 2030, sustainable development, interdependence, multidimensional crisis.

\section{INTRODUCCIÓN}

La crisis sanitaria, económica, política y social a la que nos ha abocado la pandemia de COVID-19 ha puesto de manifiesto que nos encontramos en un mundo transnacionalizado e interdependiente; en una sociedad del riesgo global (Beck, 2004) que ha generado desafíos y amenazas comunes para todas las sociedades de este sistema que llamamos planeta Tierra. Estos procesos de interconexión 
e interdependencia — propios de la edad contemporánea- han construido un mundo de comunidades de destinos solapadas (Held, 2005) que demandan acciones cooperativas y colaborativas entre Estados, para gestionar desafíos globales que afectan a todas las comunidades del planeta.

En este marco de transnacionalización, en 2015 surge la Agenda 2030 para el Desarrollo Sostenible, que se ha configurado como la apuesta más importante y ambiciosa que la comunidad internacional ha implantado para promover el desarrollo humano y sostenible a escala nacional y global. Se trata de una agenda con 17 Objetivos de Desarrollo Sostenible (ODS) y 169 metas globales. Estos abarcan una gran parte de las dimensiones que afectan al desarrollo y proponen construir un mundo próspero, justo y equitativo (Naciones Unidas, 2015). Así, la Agenda 2030 se posiciona como un marco integral de transformación de estructuras, procesos y actores que afectan al desarrollo. Por lo tanto, es posible entenderla como una apuesta radical y estructural de transformación política.

En este marco, la Agenda 2030 se presenta como une verdadera oportunidad para colocar en el centro del sistema político y económico al desarrollo sostenible. Con ello se busca dar respuesta a la crisis multidimensional en la que se encuentra la humanidad y que está poniendo en riesgo la supervivencia de millones de seres humanos y especies que habitan el planeta. Al tiempo, se trata una agenda en disputa (Martínez y Martínez, 2016) que presenta importantes potencialidades y límites para la promoción del desarrollo global. Por ello, el presente artículo pretende realizar un análisis amplio de esta agenda para comprehender las oportunidades, potencialidades y limitaciones que plantea en cuanto a la promoción del desarrollo humano y la sostenibilidad ambiental.

Partiendo de estos elementos, luego de esta breve introducción, el segundo epígrafe analiza la crisis multidimensional del desarrollo sostenible; el tercero describe las características principales de la Agenda 2030 y la conceptualiza como un intento de respuesta a las crisis mencionadas; el cuarto estudia las potencialidades y límites de la agenda; y el último epígrafe concluye con algunas reflexiones sobre la importancia de la Agenda 2030 como una apuesta política y transformadora para cambar el mundo y construir sociedades resilientes, equitativas y sostenibles. 


\section{LA CRISIS MULTIDIMENSIONAL DEL SISTEMA DE DESARROLLO}

El proceso de globalización - cuyo apogeo empieza en la década de 1990, tras la caída del Muro de Berlín- ha reconfigurado todas las dimensiones de la vida de las organizaciones humanas. Asimismo, la dinámica globalizadora ha transformado las relaciones internacionales entre Estados, al reducir el espacio de acción de los actores nacionales y al ampliar, a su vez, el ámbito de poder de los mercados y actores privados internacionales (Strange, 2001; Held, 2005; Beck, 2005).

Este proceso globalizador hace parte de la ampliación trasnacional del sistema capitalista, que ha priorizado la dimensión económica por encima de las otras dimensiones del desarrollo (Unceta, 2009). De esta forma solamente se valoran y cuantifican las actividades monetizadas, lo que promueve un concepto de crecimiento y desarrollo que se sustenta en la explotación de la naturaleza, la colonización de otros pueblos y la explotación del cuerpo y el trabajo de las personas (especialmente de las mujeres) (Herrero, 2015; Kaplan, 2002). En este marco, la mayoría de las estrategias de desarrollo y crecimiento económico (tanto de los países considerados desarrollados como en vías de desarrollo) impulsan la rápida acumulación de capital físico, financiero y humano, sin considerar que se trata de modelos basados en la degradación excesiva del capital natural, la biodiversidad y los ecosistemas (Mulligan, 2015; Naciones Unidas, 2020).

Así las cosas, la hegemonía del sistema productivo y económico globalizado y mercantilista ha enfrentado la humanidad a una crisis sistémica que afecta al ámbito social, ecológico, político y económico, como bien lo indica el texto de la Declaración a que alude el presente artículo.

Nos hemos reunido en un momento en que el desarrollo sostenible afronta inmensos desafíos. Miles de millones de nuestros ciudadanos siguen viviendo en la pobreza y privados de una vida digna. Van en aumento las desigualdades, tanto dentro de los países como entre ellos [...] Los riesgos mundiales para la salud, el aumento de la frecuencia y la intensidad de los desastres naturales, la escalada de los conflictos, el extremismo violento, el terrorismo y las consiguientes crisis humanitarias y desplazamientos forzados 
de la población amenazan con anular muchos de los avances en materia de desarrollo logrados durante los últimos decenios [...] El cambio climático es uno de los mayores retos de nuestra época y sus efectos adversos menoscaban la capacidad de todos los países para alcanzar el desarrollo sostenible [...] Peligra la supervivencia de muchas sociedades y de los sistemas de sostén biológico del planeta. (Naciones Unidas, 2015, p. 5)

Se trata, por lo tanto, de una crisis multidimensional donde se integran e interconectan distintos fenómenos sociales, políticos y ambientales, los cuales han generado vulnerabilidades que afectan a gran parte de la población del planeta. De esta forma, la evidencia empírica parece demostrar que en las últimas décadas se ha producido un incremento considerable de la desigualdad, la precarización, la violencia sistémica y la vulnerabilidad social (Organización para la Cooperación y el Desarrollo Económicos [OCDE], 2011; OCDE, 2018), al tiempo que el Estado ha ido perdiendo capacidad y poder tanto para proteger a su ciudadanía, como para intervenir y controlar los mercados financieros y económicos (Strange, 2001; Oxfam, 2018).

Por último, cabe destacar la crisis de legitimidad democrática que está impactando de manera significativa a la mayoría de las democracias liberales occidentales (Castells, 2018). La idea de que estamos en una crisis de representación se podría caracterizar como un marco de profunda desconfianza y apatía de la ciudadanía con respecto a sus gobernantes y al funcionamiento de las instituciones básicas de las democracias contemporáneas (Eberhardt, 2015).

En las últimas décadas los Estados han vivido un profundo proceso de desregulación y pérdida de poder, que ha incrementado, a su vez, el poder de los actores privados para intervenir y modificar los procesos públicos de toma de decisiones, tanto a escala nacional como global. En este marco, se extiende la percepción ciudadana de que la soberanía de los Estados se ha erosionado, y que los actores públicos se ven afectados de manera más directa por intereses privados que se encuentran fuera del ámbito democrático de representación política. Todo esto pone en peligro la legitimidad que la ciudadanía le otorga a las instituciones democráticas, la cual es fundamental para que la democracia se pueda fortalecer, expandir y consolidar. 
En definitiva, el sistema hegemónico de producción y consumo que utiliza al crecimiento económico como principal - y casi único- indicador de progreso y bienestar, ha generado un sistema que pone en peligro la estabilidad de las organizaciones humanas y la supervivencia de la naturaleza. En tal sentido, la Agenda 2030 para el Desarrollo Sostenible asume, en su texto, que es necesario un cambio cardinal de acción política para reconfigurar el sistema global y nacional, y generar así sociedades que pongan en el centro la dignidad de las personas y la protección del planeta.

\section{La Agenda 2030 para el desarRollo sostenible como UNA RESPUESTA A LA CRISIS SISTÉMICA DEL DESARROLLO}

Como explicó, la Agenda 2030 para el Desarrollo Sostenible, aprobada en 2015 por las Naciones Unidas, es claramente la apuesta más importante de la comunidad internacional para promover el desarrollo humano, asegurar la sostenibilidad ambiental y construir un sistema que proteja a las personas y "no deje a nadie atrás" (Naciones Unidas, 2015). Se trata de un plan con 17 objetivos y 169 metas que insta a "transformar el mundo", abordando de manera comprehensiva todas las políticas públicas que se desarrollan a escala local, nacional y global.

La Agenda 2030 parte de la premisa de que es necesario poner en el centro de la política a la dignidad humana, y asume que solo desde la construcción de sociedades justas, sostenibles y equitativas es posible construir la paz y la prosperidad en el planeta. Por último, la agenda configura un ámbito de actuación amplio que incorpora a todos los actores - tanto públicos como privados- en el trabajo por el desarrollo sostenible.

Una manera interesante de comprehender la Agenda 2030 es dividir sus ODS en función de cincos ejes fundamentales: personas, prosperidad, planeta, paz y partenariado ${ }^{1}$. Así, en las personas se sitúan los

1 Esta última acepción hace referencias a las alianzas globales que propone la agenda en el sentido de un "partenariado global" para el desarrollo. Según el Programa de las Naciones Unidas para el Desarrollo (PNUD), esta perspectiva remite a la promoción de la participación a través del diálogo y la negociación entre diversos actores que establecen un programa de acciones conjuntas para beneficiar a todos los actores involucrados y promover el desarrollo (PNUD, 2021). 
primeros seis objetivos, que hacen referencia a la necesidad de transformar las políticas y los procesos para luchar contra la violencia y la vulnerabilidad humana. En el segundo eje, planeta, se ubican cuatro objetivos relacionados con el cuidado de la biosfera del planeta, la producción y el consumo responsable, y la lucha contra el cambio climático. El tercer pilar, prosperidad, remite a la promoción de una economía justa, equitativa y sostenible, que le permita a todas las personas ejercer su derecho al desarrollo. Por su parte, la paz radica en el ODS número 16, mediante el cual se propone la construcción de sociedades pacíficas, justas y con instituciones sólidas y cohesionadas. Por último, el partenariado hace referencia a la necesidad de construir una alianza global para el desarrollo, en la que todos los actores -Gobiernos nacionales y locales, instituciones multilaterales, empresas, universidades, organizaciones no gubernamentales para el desarrollo (ONGD) y movimientos sociales- trabajen en conjunto para promover el desarrollo bajo el principio de responsabilidades compartidas pero diferenciadas (Naciones Unidas, 1992).

Como se observa, se trata de una propuesta política de profunda transformación que parece inspirarse en las perspectivas que abogan por concebir el desarrollo como libertad y como promoción de capacidades, para que todas las personas pueden elegir cómo quieren vivir sus vidas (Sen, 1999; Nusbaum y Sen, 2002). Por ello, la Agenda 2030 concibe la promoción de la paz en un sentido positivo, lo que implica la eliminación de toda violencia estructural (Galtung, 1996) y la configuración de sociedades que permitan la armonía del ser humano consigo mismo, con los demás y con la naturaleza.

Asimismo, la dimensión ambiental aparece transversalizada en todos los ODS, dado que, desde 2012, en los foros de las Naciones Unidas se decidió que esta dimensión iba a ser el pilar de la nueva agenda de desarrollo (Naciones Unidas, 2012). Este hecho se erige como una fortaleza fundamental de esta agenda, en tanto que la evidencia empírica y científica demuestran que el modelo económico hegemónico es incompatible con la estabilidad ambiental y el sostenimiento de los recursos de la naturaleza (Hickel, 2020; Ripple et ál., 2020; Naciones Unidas, 2020). En relación con este punto, parece necesario considerar que la consecución de las metas contenidas en los ODS supondría una transformación radical de las estructuras económicas que configuran el sistema capitalista en el ámbito local, nacional, regional y global. 
Todo esto, lleva a enfatizar la dimensión política de la Agenda 2030, en tanto que los ODS demandan acciones ambiciosas y transformadoras que, de ponerse en práctica, supondrán importantes conflictos y resistencias tanto para la práctica tradicional de la política de los Estados y sus llamados intereses nacionales" ${ }^{2,}$ como para los intereses privados de los actores hegemónicos del sistema.

De todo lo expresado es posible concluir que el potencial más importante de la Agenda 2030 es que se erige analíticamente sobre un paradigma de transformación integral, estructural, complejo y radical de la acción política de todos los actores que trabajan por (o se relacionan con) el desarrollo sostenible. En tal sentido, si esta propuesta teórica pretende trascender la esfera narrativa, parece claro que es necesario configurar procesos y políticas públicas con objetivos y prácticas que pongan en el centro al desarrollo sostenible. De igual modo, estas acciones deberán desplazar las prácticas hegemónicas que entienden que el desarrollo es asimilable al crecimiento económico y que el resto de las dimensiones son procesos secundarios e incluso prescindibles en la promoción del progreso de las sociedades humanas.

Por último, la Agenda 2030 destaca la importancia de las políticas públicas y de los gobiernos como actores centrales en las dinámicas del desarrollo. Así lo demuestra la experiencia de los países en los Exámenes Nacionales Voluntarios (VNRs, por sus siglas en inglés), donde las administraciones públicas son las principales protagonistas de los procesos de implementación de la agenda, y para esto necesitan importantes capacidades técnicas y políticas que permitan configurar un sistema de implementación, seguimiento y evaluación de políticas en el marco de los ODS (Naciones Unidas, 2019a).

2 En las agendas de política exterior parece existir una tendencia, de corte realista, a dividir los intereses de los Estados en dos agendas: una agenda dura, que constituye el núcleo central de los llamados intereses nacionales y que se compone fundamentalmente de temas económicos, energéticos y de seguridad. En un segundo nivel se ubicaría la agenda blanda, que incorpora aquellos elementos relativos a la promoción del desarrollo, la defensa de los derechos humanos, las cuestiones de género y la sostenibilidad ambiental (Santander y Millán, 2014). 


\section{Potencialidades y limitaciones de la Agenda 2030 para EL DESARROLLO SOSTENIBLE}

El presente epígrafe pretende analizar las potencialidades y limitaciones de la Agenda 2030 con base en el diagnóstico de crisis multidimensional descrito en los apartados anteriores. Teniendo en cuenta las características de este artículo de investigación, se trata de una sección que no pretende —ni puede - agotar esta temática, pero sí mencionar, de manera sucinta y analítica, los elementos principales que caracterizan a esta agenda de desarrollo.

\subsection{Potencialidades y fortalezas de la Agenda 2030}

\subsubsection{Visión COSMOPOLITA PARA Gestionar LOS BIENES Y MALES PÚblicos GLOBALES}

En primer lugar, la Agenda 2030 establece la importancia de la interdependencia y la interconexión entre las sociedades actuales al asumir que existen bienes (y males) públicos globales, cuyas externalidades han traspasado las fronteras nacionales $\mathrm{y}$, por lo tanto, demandan una acción colectiva entre países para poder dar respuestas a estas amenazas a la seguridad humana (Kaul et ál., 1999; Beck, 2004; Held, 2010).

Por ejemplo, la pandemia de coronavirus — que ha afectado a una gran parte de las sociedades del planeta-demuestra empíricamente que nos encontramos en un momento de emergencia trasnacional. Esto evidencia que el sistema está construido sobre sociedades profundamente interdependientes y asimétricas, donde se generan riesgos que trascienden las fronteras nacionales y se convierten en verdaderas amenazas para la supervivencia de las sociedades humanas (Millán y Santander, 2020). Así, los problemas del desarrollo se han transnacionalizado (Millán, 2012) y demandan una acción cooperativa entre Estados y administraciones públicas, que superen las fronteras nacionales y generen respuestas cosmopolitas.

En este sentido, la mirada cosmopolita permite comprehender, de manera más asertiva, la realidad globalizada del desarrollo sustentable y las características de los Estados posnacionales (Habermas, 2000), cuyas fronteras son cada vez más porosas y difusas. La globalización ha supuesto una transformación fundamental de la configuración del 
Estado nación. Esto tiene consecuencias radicales para la teoría política del desarrollo, dado que las prácticas y procesos que se implementan en el marco de los ODS deben incorporar necesariamente una perspectiva transnacional. De esta argumentación se deriva la propuesta de que los actores políticos adopten una mirada cosmopolita que les permita comprehender que los desafíos de desarrollo que se generan en sus propios territorios están imbricados con los espacios globales y que, por lo tanto, el nacionalismo metodológico ${ }^{3}$ (Beck, 2005) es insuficiente para analizar y gestionar los conflictos y problemas sociales.

En este marco, la Agenda 2030 se establece con un carácter universal, dado que demanda una garantía global de respeto a los derechos humanos, al tiempo que establece objetivos y metas que deben ser implementadas por todos los países (independientemente de su nivel de desarrollo). Además, el texto de la Declaración de Desarrollo Sostenible hace mención explícita a la necesidad de desplegar estrategias trasnacionales para gestionar bienes públicos globales, como la lucha contra el cambio climático, la gestión de las migraciones internacionales o la protección de los derechos humanos, entre otros (Naciones Unidas, 2015). En este sentido, la Agenda 2030 parece asumir, en su texto y espíritu, la necesidad de abordar de manera conjunta esta nueva realidad, que supone riesgos globales que trascienden y desbordan las tradicionales fronteras nacionales.

\subsubsection{Perspectiva transversal de la coherencia de políticas para el DESARROLLO SOSTENIBLE (CPDS)}

Un segundo elemento extremadamente sugerente en la Agenda 2030 es su visión amplia y multidimensional, que trasciende la tecnocrática y restringida de agendas anteriores y que plantea transformar y reestructurar todas las políticas públicas que afectan al desarrollo humano sostenible. Se trata de una agenda que parte del paradigma de la CPDS (Millán, 2020), en tanto asume la visión del desarrollo como un espacio de trabajo para todos los actores y una

3 El nacionalismo metodológico se basa en la idea de que para comprender los problemas y desafíos a los que se enfrentan las sociedades es suficiente analizar los procesos nacionales que se desarrollan al interior de un Estado, lo que invisibiliza la importancia de los procesos trasnacionales tanto en la comprensión de los desafíos de desarrollo, como en la búsqueda de soluciones a estos (Beck, 2005). 
transformación radical de todas las políticas públicas. Cabe destacar que el concepto de CPDS debe entenderse como la integración de la perspectiva de desarrollo sostenible en el diagnóstico, diseño, implementación y evaluación de todas las políticas públicas de un país. Esto significa transversalizar el trabajo por el desarrollo en toda la acción gubernamental (Millán, 2014; OCDE, 2015).

Es importante señalar que la CPDS posee una dimensión política que implica que, para transformar radicalmente la acción de los Gobiernos en sintonía con el desarrollo sostenible, es necesario un profundo compromiso político por parte los actores de poder público. En este sentido, el hecho de que la agenda parta de un paradigma transversal, complejo y multidimensional, se puede plantear como como un punto de inflexión en el trabajo por el desarrollo y una indudable oportunidad para poner en el centro de la acción pública el cuidado de la vida y la sostenibilidad, para transformar así a los actores y políticas que configuran la estructura de las sociedades. Por lo tanto, la Agenda 2030 es consistente con la propuesta doctrinaria de la CPDS, ya que parte de un paradigma de transformación integral y radical de la acción política de un gobierno y de las relaciones de este con los actores trasnacionales.

\subsubsection{Visión MULTIACTOR Y MULTINIVEL PARA GESTIONAR LOS DESAFÍOS DEL DESARROLLO}

En tercer lugar, la agenda parte de la premisa que para avanzar en la consecución de los ODS es necesario incorporar a todos los niveles de las administraciones públicas que tienen responsabilidad e impactos sobre los procesos de sostenibilidad, equidad y seguridad humana. Esto supone una apuesta clara por el ámbito local, destacada especialmente en el ODS número 11, en el cual se alude directamente a las ciudades sostenibles. En tal sentido, el hecho de que las administraciones sean consideradas institucionales cardinales para la promoción del desarrollo sostenible es una gran fortaleza, dado que la mayor parte de las metas contenidas en los ODS no se podrían alcanzar fuera del ámbito local (Gobierno de España, 2020). Además, la incorporación protagónica de Gobiernos descentralizados permite avanzar en la localización de la agenda, el desarrollo de estrategias específicas, la adaptación de los objetivos a las estructuras políticas y económicas particulares, y la sensibilización de la 
ciudadanía que se encuentra más cercana a estos niveles de gobierno. Esto supone una importante fortaleza de la agenda, dado que genera oportunidades para revitalizar y actualizar las estrategias de cada territorio, estimulando la identificación de retos comunes, promoviendo la cooperación internacional con otros territorios e impulsando una visión amplia e inclusiva del desarrollo sostenible (Gobierno de España, 2020).

Asimismo, y como se explicó en epígrafes anteriores, en la Declaración de Desarrollo Sostenible es esencial la necesidad de establecer alianzas con todos los actores de la sociedad civil, incluidas las empresas, universidades, movimientos sociales, ONGD o cooperativas, por solo mencionar algunos ejemplos. Cabe destacar que la propia experiencia de las políticas y prácticas de desarrollo nos enseñan que estos actores desempeñan funciones esenciales y complementarias, y que solo desde un trabajo cooperativo y abierto en concordancia con la sociedad civil es posible construir instituciones sólidas y democráticas que protejan a la ciudadanía y promuevan la sostenibilidad. Esta propuesta de la Agenda 2030 — que se expresa claramente en su ODS número 17 de creación de una alianza global de desarrollo- ha generado una participación de la sociedad civil tanto en las consultas públicas para la configuración de la Agenda 2030 (2013-2015), como en la mayor parte de las estrategias de implementación de los ODS que están desarrollando los países (Naciones Unidas, 2019b; Naciones Unidas, 2020c), lo que aporta riqueza, amplitud y eficacia a dichas estrategias.

En síntesis, la Agenda 2030 presenta una oportunidad crucial para asumir integralmente que el desarrollo sustentable es un trabajo común y cooperativo que debe involucrar a todos los actores de la sociedad, al tiempo de estimular la creatividad, la apropiación y el compromiso de las instituciones.

\subsubsection{LA APUESTA POR LO PÚBLICO Y LA PROMOCIÓN DE LA DEMOCRACIA}

Tanto la Declaración como los propios ODS remiten a la necesidad ineludible de fortalecer las políticas públicas para promover la cohesión social, la lucha contra la desigualdad y el impulso del desarrollo sostenible. Esto alude, necesariamente, a una perspectiva política que coloca en primer término al papel de la acción pública y del Estado. En este sentido, es necesario avanzar en el fortalecimiento de los actores públicos, con el objetivo de que tengan 
capacidades para desarrollar políticas que protejan a la población de las amenazas y riesgos a los que se enfrentan las sociedades, como es el caso actual de la pandemia de COVID-19. Al tiempo, las teorías contemporáneas de la democracia proponen que este sistema debe trascender los elementos formales y procedimentales que la configuran, dado que, aunque son procesos fundamentales, parecen no ser condición suficiente para sostener y legitimar este sistema de organización política (Held, 2005; Levitsky y Ziblatt, 2018; Spitz, 2019). En tal sentido, la promesa de la democracia radica en promover un sistema justo, equitativo y sostenible para que todas las personas puedan ejercer sus derechos con plena conciencia y ser libres para elegir la vida que desean. En contraposición, la desigualdad, la pobreza y la exclusión deslegitiman las instituciones democráticas y reducen la confianza de la ciudadanía en este régimen de gobierno (Heller, 1985; Vilas, 1994). En suma, la democracia es más que un sistema formal de representación política y debe ser comprehendido como un espacio de equidad, cohesión y protección para la ciudadanía.

En este marco, se puede entender la Agenda 2030 como una verdadera apuesta por la profundización democrática, ya que plantea promover sociedades más justas, sostenibles e inclusivas que desarrollen políticas públicas que protejan a la ciudadanía de las privaciones y la opresión. Así, todos los ODS pueden ser leídos en clave de profundización democrática, en tanto pretenden promover los derechos de las personas.

Los diez primeros ODS se relacionan directamente con mejorar las condiciones de vida, el acceso a los derechos económicos y sociales y la lucha contra la desigualdad; los ODS relacionados con el medioambiente $(11,12,13,14$ y 15) se orientan a que la ciudadanía pueda ejercer sus libertades en condiciones psicofísicas adecuadas; y, por último, los ODS 16 y 17 buscan generar instituciones, pacíficas, sólidas y cooperativas, elementos fundamentales en las sociedades democráticas.

Por lo tanto, aunque la Agenda 2030 menciona la democracia de manera explícita una vez (Naciones Unidas, 2015:4), es posible concluir que la propuesta transversal de construcción de sociedades pacíficas y sostenibles es un elemento cardinal de la Declaración y de los ODS. 


\subsection{Limitaciones de la Agenda 2030: despolitización, TECNOCRACIA Y ESTRUCTURAS DE PODER}

Como se ha explicado, las limitaciones de la Agenda 2030 se relacionan principalmente con la falta de problematización y visibilización de los conflictos de poder que conlleva poner en el centro el desarrollo sostenible. A juicio de este trabajo, son tres los elementos para destacar en este sentido:

\subsubsection{La falta de obligatoriedad de la Agenda 2030}

Tradicionalmente las agendas globales y nacionales que se orientan a la promoción del desarrollo global —más relacionadas, hasta el 2015, con el ámbito de la cooperación internacional—se caracterizan por su naturaleza discrecional y no vinculante, dado que se trata de actividades que permiten fortalecer y promover el desarrollo. No obstante, deben estar acordes con las estrategias, visiones e intereses particulares de los países que forman parte del sistema. De hecho, se suele argumentar que, si estas políticas fueran imperativas, no se pondrían en práctica con éxito, dado que implicarían una obligatoriedad que los Estados no están dispuestos a asumir. Ahora bien, el hecho de que la Agenda 2030 esté dotada de un carácter voluntario y discrecional, amplía el riesgo de que los Gobiernos no asuman esta agenda como un imperativo político para desarrollar programas de desarrollo, sino como una elección voluntaria que depende las necesidades y visiones de cada administración en particular.

Así, las políticas discrecionales estimulan relaciones asimétricas entre países, al tiempo que incrementan la aleatoriedad y la impredecibilidad, lo que genera problemas de eficacia en la implementación de las políticas públicas (Santander, 2020). A diferencia de otras agendas internacionales, que sí son ineludibles (como las relativas a la regulación del comercio internacional bajo la OMC), el carácter no vinculante de la Agenda 2030 pareciera colocarla en una posición secundaria y menos relevantes que las agendas económicas y de seguridad.

En suma, aun cuando la agenda incorpora importantes potencialidades normativas e ideacionales que pueden tener impactos destacados en los factores materiales e institucionales de los Gobiernos (Sanahuja, 2016), este carácter discrecional supone limitaciones para 
que trascienda el ámbito narrativo y despliegue una capacidad real de transformación política.

\subsubsection{LOS RIESGOS DE UNA INTERPRETACIÓN TECNOCRÁtICA DE IMPLEMENTACIÓN de la Agenda 2030}

Como se mencionó, la Agenda 2030 se puede concebir como un hecho normativo en disputa (Martínez y Martínez, 2016), ya que es posible interpretarla como una apuesta integral y transformadora de cambio radical en las políticas públicas; o bien como una agenda tecnocrática de implementación de mecanismos y recursos para realizar reformas puntuales en algunas políticas específicas.

En el primer caso se asume que la agenda posee una dimensión eminentemente política y que el trabajo por el desarrollo se orienta a gestionar estructuras de poder. En tal sentido, es fundamental el compromiso político de los Gobiernos, así como las instancias de negociación, la posición de las instituciones y las presiones e intereses de los actores privados. En el caso contrario, se monopoliza una visión tecnocrática donde se intentan promover espacios de oportunidad para que los diferentes Gobiernos simplemente mencionen o potencien aquellas políticas que encajan (Gutiérrez, 2020) en la Agenda, pero sin asumir el compromiso de transformación y conflicto que supone implementar transversalmente políticas públicas de desarrollo sostenible.

Esta visión de corte tecnocrático y despolitizado (Santander, 2020) parece orientarse a invisibilizar las relaciones de poder y los conflictos que necesariamente van a surgir si se pretende implementar en toda su extensión y amplitud la Agenda 2030. En este sentido, la narrativa que acompaña tanto el texto de la Declaración como a los propios ODS asume que es perfectamente posible promover el crecimiento del sistema capitalista al tiempo que se avanza en equidad, inclusión social, protección de las personas y sostenibilidad ambiental (Millán, 2020). No obstante, existe suficiente evidencia empírica para demostrar que los procesos capitalistas que ponen en el centro el crecimiento económico son contradictorios con los elementos básicos del desarrollo sostenible, como son la redistribución de la riqueza (Spitz, 2019), la sostenibilidad ambiental (Naciones Unidas, 2019c), la igualdad de género (Pérez Orozco, 2014) e, incluso, la calidad democrática (Ferrajoli, 2005). 
En este sentido, al evitar mencionar las contradicciones entre la perspectiva del desarrollo humano sostenible y las características básicas del sistema capitalista, la Agenda 2030 parece invisibilizar el verdadero límite para la construcción de un mundo más equitativo y sostenible, que es el propio sistema económico y político sobre el que se erigen la mayor parte de las sociedades contemporáneas.

En síntesis, la agenda puede exponer límites relacionados principalmente con una interpretación tecnocrática y despolitizada, que no asume los conflictos políticos involucrados en la transformación radical de las políticas públicas. Así, el carácter no vinculante de la agenda conlleva que los Gobiernos pueden concebirla como un espacio secundario para la transformación política, que se expresa principalmente en el ámbito narrativo. Además, esta perspectiva puede estimular programas y procesos que tiendan a reproducir las prácticas ya existentes, aunque incorporando pequeñas reformas que no acarrean una transformación política real, en coherencia con el desarrollo sostenible. Si esta es la interpretación que finalmente se adopta de la Agenda 2030, esta perderá sus mayores potencialidades en términos de integralidad y multidimensionalidad, y se convertirá en un hecho narrativo sugerente, pero sin capacidad real de promover los cambios para "transformar nuestro mundo y no dejar a nadie atrás".

\section{Reflexiones finales}

Desde la perspectiva de la teoría y estudios de desarrollo, el balance de la propuesta integral que hace la Agenda 2030 es indudablemente positivo. En efecto, se trata de una estrategia que asume la necesidad de promover una visión amplia, compleja y multidimensional que coloque en el centro de la acción política la sostenibilidad y la seguridad humana. Además, esta agenda integra la realidad transnacionalizada y cosmopolita del sistema contemporáneo y propone alianzas que estimulen soluciones cooperativas y compartidas a los desafíos globales que actualmente enfrenta la humanidad.

Cabe destacar el potencial de la Agenda 2030 para erigirse como una respuesta integral a las diversas crisis que afectan las sociedades y que ponen en peligro la propia supervivencia de millones de seres humanos y criaturas que habitan nuestro planeta. La crisis 
sanitaria, económica, social y política derivada de la pandemia global de la COVID-19 es solo una evidencia de la sociedad del riesgo global que el sistema productivo y económico ha generado en las organizaciones humanas.

Sin embargo, la evidencia empírica no parece demostrar que las estructuras de poder y las prácticas políticas se estén transformando en clave con el desarrollo sostenible, como lo proponen los ODS (Naciones Unidas, 2020). La propia gestión de la pandemia, donde han primado las lógicas competitivas y nacionalistas, la falta de compromiso político real con la lucha contra el cambio climático, la crisis de refugio y asilo para millones de personas que no encuentran países de acogida, o los discursos nacionalistas y autoritarios, son solo ejemplos de cómo el mundo se sigue constituyendo sobre lógicas realistas y mercantilistas. Aquí se comprehende el sistema internacional como un dilema de suma cero, donde cada Estado debe procurar la optimización de su poder y recursos aún a costa de profundizar la vulnerabilidad de otras sociedades y países.

En este sentido, es ineludible abogar por que la Agenda 2030 se transforme en una verdadera apuesta por el desarrollo sostenible, la seguridad humana, la paz y la democracia. Con este objeto se vuelve necesario repolitizar esta agenda y asumir los conflictos en las estructuras de poder que supone la apuesta por colocar en el centro del sistema político el desarrollo sostenible.

En definitiva, solo desde una posición política de transformación de las relaciones de poder, y asumiendo la dimensión conflictiva de los procesos de desarrollo, se podrá impulsar la reconstrucción de sociedades resilientes y seguras, que protejan a los seres humanos, generen procesos armónicos con la naturaleza y puedan, efectivamente, hacer realidad la promesa del desarrollo de no dejar a nadie atrás.

\section{REFERENCIAS}

Beck, U. (2004). La sociedad del riesgo global. Paidós.

Beck, U. (2005). La mirada cosmopolita o la guerra es la paz. Paidós.

Castells, M. (2018). Ruptura: la crisis de la democracia liberal. Editorial Alianza.

Eberhardt, M. L. (2015). Democracias representativas en crisis. Democracia participativa y mecanismos de participación ciudadana como opción. Araucaria. Revista Iberoamericana de Filosofía, Política y Humanidades, 17(33), 83-106. 


\section{ANÁLISIS \\ JURÍDICO-POLÍTICO}

Ferrajoli, L. (2005). La crisis de la democracia en la era de la globalización. Anales de la Cátedra Francisco Suárez, 39, 37-51.

Galtung, J. (1996). Peace and conflict, development and civilization. International Peace Research Institute; Sage Publications.

Gobierno de España. (2020). Guía para la localización de la Agenda 2030. Vicepresidencia Segunda del Gobierno. https: / / bit.ly/3xR4cXP

Gutiérrez, J. (2020). La Agenda 2030 en Euskadi: ¿compromiso o apariencia? Dossier Galde: Los ODS y la Agenda 2030 a debate, (28), 26-28. https: / / bit.ly /3hCRbuZ

Habermas, J. (2000). La constelación posnacional: ensayos politicos. Paidós.

Herrero, Y. (2015). Apuntes introductorios sobre ecofeminismo. Centro de documentación HEGOA. https: / / bit.ly /36DIPwM

Heller, H. (1985). Escritos políticos. Alianza Universidad.

Held, D. (2001). Modelos de democracia. Editorial Alianza.

Held, D. (2005). Un pacto global. Editorial Taurus.

Held, D. (2010). Cosmopolitanism. Ideals and realities. Polity Press.

Hickel, J. (2020). The sustainable development index: Measuring the ecological efficiency of human development in the Anthropocene. Ecological Economics, 167, 106331. https: / / doi.org/10.1016/j.ecolecon.2019.05.011

Kaplan, M. (2002). Estado y globalización. Instituto de Investigaciones Jurídicas, UNAM.

Kaul, I., Grunberg, I. y Stern, M. (1999). Global Public Goods: International Cooperation in the 21st Century. Oxford University Press. https: / / doi.org/10.1093/ 0195130529.001.0001

Levitsky, S. y Ziblatt, D. (2018). Cómo mueren las democracias. Ariel Editorial.

Martínez, P. y Martínez, I. (2016). La Agenda 2030: ¿cambiar el mundo sin cambiar la distribución del poder? Lan Harremanak: Revista de relaciones laborales, 33, 73-102.

Millán, N. (2012). Transnacionalización del desarrollo y coherencia de políticas: un análisis de los casos de España y Suecia [tesis doctoral, Universidad Complutense de Madrid]. Repositorio institucional UCM. https: / / bit.ly / 2UIDmCv

Millán, N. (2014). Reflexiones para el estudio de la coherencia de políticas para el desarrollo. Papeles 2015 y más, 17. https: / / bit.ly / 3yUS73L

Millán, N. (2020). La coherencia de políticas en el marco de la Agenda 2030 de Desarrollo Sostenible. Revista Española de Desarrollo y Cooperación, (46), 69-78.

Millán, N. y Santander, G. (2020). El virus cosmopolita, lecciones de la COVID-19 para la reconfiguración del Estado-Nación y la gobernanza global. Geopolitica(s) Revista de estudios sobre espacio y poder, (11) 251-263.

Mulligan, M. (2015). An introduction to sustainability: environmental, social, and personal perspectives. Routledge.

Naciones Unidas. (2012). Cumbre de la Tierra. https: / / bit.ly/3kjSnFp

Naciones Unidas. (2015). Declaración de Desarrollo Sostenible. https://bit.ly/2UL$4 \mathrm{qAK}$

Naciones Unidas. Programa para el medioambiente. (2019a). 2019. Voluntary National Rewiews. Synthesis Report. https: / / bit.ly/36w6Ypj 
Naciones Unidas. (2019b). Civil society participation in the implementation of the 2030 Agenda for Sustainable Development. https: / / bit.ly /3kjSX63

Naciones Unidas. (2019c). Global Sustainable Development Report 2019. The Future is Now: Science for Achieving Sustainable Development. https: / bit.ly/3hCwkrT

Naciones Unidas. (2020, 1 de diciembre). Informe sobre la Brecha de Emisiones 2020. https: / / bit.ly/3yU55i9

Nusbaum, M. y Sen, A. (1998). La Calidad de Vida. Fondo de Cultura Económica.

Organización para la Cooperación y el Desarrollo Económicos (OCDE). (2011). Divided We Stand: Why Inequality Keeps Rising. OCDE Publishing.

Organización para la Cooperación y el Desarrollo Económicos (OCDE). (2015). Framework for policy coherence for sustainable development (PCDS). OCDE Publishing. https: / / bit.ly/2VzmTAJ

Organización para la Cooperación y el Desarrollo Económicos (OCDE). (2018). Desigualdad de ingresos: la brecha entre ricos y pobres. OCDE Publishing. https: / bit.ly/3B04HRa

Organización Internacional del Trabajo (OIT). (2020). Perspectivas sociales y del empleo en el mundo. https: / / bit.ly/2UDMfNv

Oxfam (2018): Premiar el trabajo, no la riqueza. Para poner fin a la crisis de desigualdad, debemos construir una economía para los trabajadores, no para los ricos y poderosos. https: / / bit.ly /3wDgHV1

Programa de las Naciones Unidas para el Desarrollo (PNUD). (2021). UNDP Our Partners https: / / www.undp.org/ partners

Pérez Orozco, A. (2014). Subversión feminista de la economía. Aportes para un debate sobre el conflicto capital/vida. Madrid: Traficantes de Sueños. Papeles del CEIC. International Journal on Collective Identity Research, (1), 1-4.

Ripple, W., Wolf, C., Newsome, T., Barnard, P. y Moomaw, W. (2020). World Scientists' Warning of a Climate Emergency. BioScience, 70(1), 8-12.

Sanahuja, J. A. (2016). La Agenda 2030 de desarrollo sostenible. Gaceta sindical: reflexión y debate, 26, 205-221.

Santander, G. y Millán, N. (2014). La política diplomática española y la defensa de los derechos humanos. Cuadernos 2015 y más, 3. https: / / bit.ly/3r4OA08

Santander, G. (2020). Cooperación y conflicto en la Agenda 2030: ¿una relación desequilibrada? Iberoamerican Journal of Development Studies, (10), en prensa.

Sen, A. (1999). Desarrollo y Libertad. Editorial Planeta.

Spitz, J. (2019). El capitalismo democrático: ¿el fin de una excepción histórica? Nueva Sociedad, (282), 69- 84.

Strange, S. (2001). La retirada del Estado. La difusión del poder en la economía mundial. Icaria.

Unceta, K. (2009). Desarrollo, subdesarrollo, maldesarrollo y postdesarrollo: una mirada transdisciplinar sobre el debate y sus implicaciones. Carta Latinoamericana, (7), 1-34.

Vilas, C. (1994). Mercado, estados y revoluciones: Centroamérica 1950-1990. Universidad Nacional Autónoma de México. 
